\title{
Treatment outcomes of hepatectomy for liver metastases of gastric cancer diagnosed using contrast-enhanced magnetic resonance imaging
}

\author{
Taichi Tatsubayashi $^{1} \cdot$ Yutaka Tanizawa $^{1} \cdot$ Yuichiro Miki $^{2} \cdot$ Masanori Tokunaga $^{1} \cdot$ \\ Etsuro Bando $^{1}$ - Taiichi Kawamura ${ }^{1}$ - Teiichi Sugiura ${ }^{3}$ - Yusuke Kinugasa ${ }^{3}$. \\ Katsuhiko Uesaka $^{3} \cdot$ Masanori Terashima $^{1}$
}

Received: 19 November 2015/Accepted: 23 April 2016/Published online: 7 May 2016

(c) The International Gastric Cancer Association and The Japanese Gastric Cancer Association 2016

\begin{abstract}
Background Chemotherapy is the standard treatment for liver metastases of gastric cancer (LMGC). Hepatectomy for LMGC reportedly has a 5-year survival rate of 13-37\%; however, its significance has not been established. At our hospital, hepatectomy is performed for patients with three or fewer metastases diagnosed using contrast-enhanced magnetic resonance imaging (MRI). To identify the ideal patient subpopulation for resection, we retrospectively analyzed treatment outcomes in patients with LMGC who underwent hepatectomy.

Methods Clinicopathological factors affecting survival were explored using univariate and multivariate analyses in 28 patients who underwent hepatectomy for LMGC diagnosed using contrast-enhanced MRI between December 2004 and October 2014.

Results The study included 23 men and 5 women with a median age of 72 years. Metastases were synchronous in 15 patients and metachronous in 13 patients. The median overall survival time was 49 months, with a 5-year survival rate of $32 \%$. Univariate analysis revealed that overall survival time was shorter in the presence of the following factors: age $\geq 70$ years $(p=0.030)$, synchronous liver metastases $(p=0.017)$, and presence of postoperative
\end{abstract}

Masanori Terashima

m.terashima@scchr.jp

1 Division of Gastric Surgery, Shizuoka Cancer Center, 1007 Shimonagakubo Nagaizumi-cho, Sunto-gun, Shizuoka 411-8777, Japan

2 Department of Surgical Oncology Graduate School of Medicine, Osaka City University, Osaka, Japan

3 Division of Gastrointestinal Surgery, Shizuoka Cancer Center, Shizuoka, Japan complications $(p=0.042)$. In patients with metachronous liver metastases, the post-resection 5-year survival rate was $59 \%$.

Conclusions The 5-year survival rate was $32 \%$ in patients who underwent hepatectomy for LMGC according to our criteria, suggesting that hepatectomy is an important treatment if indications are on the basis of contrast-enhanced MRI. Therefore, active resection should be considered, particularly for patients with metachronous liver metastases.

Keywords Gastric cancer - Liver metastasis .

Hepatectomy

\section{Introduction}

The most common sites of metastatic recurrence of gastric cancer are the peritoneum and lymph nodes, and the most common site of hematogenous metastasis is the liver [1-3]. This distinction is also true for recurrence after radical resection of gastric cancer; therefore, resection is rarely indicated for these lesions. Systemic chemotherapy is the standard treatment for patients with distant metastasis or recurrence; however, the reported median survival time (MST) is only 10-14 months [4, 5]. In contrast for patients with peritoneal or distant lymph node metastases, resection is indicated for some patients with liver metastases. Standard resection is performed in cases of resectable liver metastases of colorectal cancer [6], and 5-year survival rates exceeding $50 \%$ have been reported $[7,8]$. Because liver metastases of gastric cancer (LMGC) are often multifocal at the time of diagnosis [9], hepatectomy is not commonly recommended. Nevertheless, excellent outcomes of hepatectomy for LMGC have recently been 
reported from several high-volume centers, with a 5-year survival rate of 13-37\% and MST of 11-34 months [1015]. However, the majority of these reports were based on single-center retrospective analyses of relatively small sample sizes collected over long periods and without clearly defining the indications for resection. Furthermore, diagnostic modalities of liver metastases were not precisely described in these reports. The efficacy of contrast-enhanced magnetic resonance imaging (MRI) using a liverspecific contrast agent for the diagnosis of liver metastasis has been recently reported [16].

To identify the optimal target subpopulation for resection in patients with LMGC, we retrospectively analyzed the treatment outcomes of hepatectomies performed at our hospital based on modern diagnostic technology.

\section{Methods}

\section{Patients}

The data for 28 patients who underwent hepatectomy for LMGC were retrospectively analyzed at the Shizuoka Cancer Center between December 2004 and October 2014. All patients were diagnosed by contrast-enhanced MRI using a liver-specific contrast agent. Liver resection was indicated for patients with LMGC if they met the following inclusion criteria: (1) no more than three metastases, (2) no noncurative factors other than liver metastases, (3) eligible for radical surgical resection, and (4) good general health condition. The liver metastasis was diagnosed by multiple imaging modalities such as contrast-enhanced computed tomography (CT), ultrasound sonography, and contrastenhanced MRI using liver-specific contrast agent. With regard to the liver-specific contrast agent, we used superparamagnetic iron oxide until 2008 and then gadolinium ethoxybenzyl-diethylenetriamine pentaacetic acid after 2009.

\section{Data collection}

Clinicopathological data were collected from databases prospectively registered into electronic medical records. Histopathological descriptions were handled in accordance with the Japanese Classification of Gastric Carcinoma (Third English Edition) [17]. Liver metastases already present when gastric cancer had been diagnosed were defined as synchronous metastases, whereas metastases that occurred in the liver after the curative resection of primary gastric tumors were defined as metachronous metastases. Postoperative complications were defined as those of grade II or above according to the Clavien-Dindo classification [18]. Data collection and analysis were approved by the institutional review board of the Shizuoka Cancer Center.

\section{Statistical analysis}

Fisher's exact test and the Mann-Whitney $U$ test were used to compare the two groups. Survival curves were created using the Kaplan-Meier method. Univariate and multivariate analyses of factors affecting survival time were performed using the Cox proportional hazards model.

Statistical analysis was conducted using EZR version 1.24 (Saitama Medical Center, Jichi Medical University, Saitama, Japan) [19], and $p$ values less than $5 \%$ were considered to indicate a statistically significant difference.

\section{Results}

\section{Patient characteristics}

The clinicopathological characteristics of the 28 patients are presented in Table 1 . The male-to-female ratio was 4.6:1. In the synchronous liver metastasis cases, the primary gastric tumor was resected at the same time as the

Table 1 Clinicopathological characteristics of 28 patients who underwent hepatectomy for liver metastases of gastric cancer

\begin{tabular}{ll}
\hline Variables & $72(39-86)$ \\
\hline Age at the time of hepatectomy (years) & \\
Sex & 23 \\
Male & 5 \\
Female & \\
Timing of liver metastases & 13 \\
Metachronous & 15 \\
Synchronous & \\
Number of liver metastases & 20 \\
1 & 7 \\
2 & 1 \\
3 & $24.5(6-95)$ \\
Maximum size of liver metastases (mm) & \\
Maximum size of primary tumor (mm) & \\
Pathological T classification of the primary tumor (pT) & $56.5(0-122)$ \\
pT1, pT2 & 8 \\
pT3, pT4 & 20 \\
Pathological N classification of the primary tumor (pN) \\
pN0 \\
pN1, pN2, pN3 & 3 \\
Histological differentiation of primary tumor & 25 \\
Differentiated & 22 \\
Undifferentiated & 6 \\
\hline
\end{tabular}

${ }^{a}$ Median (range) 
liver, whereas in the metachronous liver metastasis cases, the primary gastric tumor was resected during the initial surgery. The median time from gastrectomy to hepatectomy in the 13 patients with metachronous liver metastases was 19 months (range, 8-38 months). Chemotherapy was selected for initially unresectable liver metastases, and hepatectomy was then performed for 3 patients ( 2 synchronous and 1 metachronous case) after a reduction in metastases was observed.

\section{Comparison of surgical outcomes between synchronous and metachronous liver metastases}

The amount of intraoperative blood loss and the proportion of patients who received an intraoperative blood transfusion were greater in the synchronous liver metastasis group, although the difference was not statistically significant (Table 2). Postoperative complications in patients with complication grade II or higher (determined as per the Clavien-Dindo classification) occurred in ten synchronous patients and one metachronous patient, indicating the occurrence of significantly more complications in the former $(p=0.002)$. Anastomotic leakage occurred in two patients in the synchronous liver metastases group; however, hepatectomy-induced complications, such as bile leakage and cholangitis, were more common (four patients). All patients were macroscopically and microscopically negative for surgical margins. The number of patients with multiple metastases was greater in the synchronous liver metastasis group $(p=0.038)$. The length of postoperative hospital stay was significantly prolonged in the synchronous liver metastasis group $(p=0.003)$. No inhospital deaths occurred; all patients were safely discharged.

\section{Postoperative treatment}

Twelve patients $(43 \%)$ underwent postoperative adjuvant chemotherapy after hepatectomy. The chemotherapy regimen was $\mathrm{S}-1$ in 10 patients, S-1 + cisplatin in 1 patient, and capecitabine + cisplatin + trastuzumab in 1 patient.

\section{Survival and prognostic factors}

The median follow-up period after hepatectomy was 26 months, and the median overall survival time was 49 months, with a 5-year survival rate and relapse-free survival rate of $32 \%$ and $29 \%$, respectively (Figs. 1, 2).

Univariate analysis of factors affecting survival time revealed that age 70 years or older $(p=0.030)$, synchronous liver metastases $(p=0.017)$, and postoperative complications $(p=0.042)$ resulted in a significantly poor survival outcome (Table 3). The 5-year survival rate after resection in the metachronous liver metastasis group was $59 \%$ (Fig. 3).

The variables that showed significant differences in the univariate analysis-age, timing of liver metastases, and postoperative complications-were subjected to multivariate analysis using the number of liver metastases and intraoperative blood transfusion amount, which showed differences of $p<0.1$ in the univariate analysis, as covariates. None of the analyzed variables was identified as an independent prognostic factor.

\section{Recurrence pattern}

Recurrence occurred in 17 patients (61\%) during the follow-up period, 15 (83\%) of whom experienced recurrence in the liver remnant. In addition, brain and lymph node metastases occurred in 1 patient each. Eight patients underwent chemotherapy for recurrence, 6 received best supportive care, and 3 underwent repeat hepatectomy for recurrence in the liver remnant. Of the 3 patients who underwent repeat hepatectomy, 2 survived without recurrence for more than 4 years from the initial hepatectomy, and the remaining (1) patient experienced recurrence in the abdominal wall and ribs 10 months after repeat hepatectomy and died 19 months after surgery.

\section{Discussion}

Good survival outcomes of hepatectomy were obtained with MST of 49 months and a 5-year survival rate of $32 \%$ in patients with LMGC who were diagnosed by contrastenhanced magnetic resonance imaging (MRI) using liverspecific contrast agents. These results were relatively better than those of the previous reports, which included MST of 11-34 months and a 5-year survival rate of $13-37 \%$. In previous studies, solitary metastasis had been reported as an independent better prognostic factor [11-13], resulting in hepatectomy generally being considered only for patients with a solitary liver metastasis.

Oki et al. recently reported that solitary metastasis was an independent prognostic factor in a large-scale multiinstitutional cohort study [20]. Patients with multiple liver metastases are generally poor candidates for hepatectomy. In the recent Japanese Clinical Oncology Group (JCOG) trial, which investigated the role of volume reduction surgery in patients with a single noncurable factor, it was reported that the presence of two to four liver metastases, distant lymph node metastasis, and peritoneal metastasis were factors associated with noncurable disease [21]. However, Kinoshita et al. had reported that survival was better in patients with fewer than three liver metastases in a 
Table 2 Operative details and perioperative outcomes of hepatectomy

\begin{tabular}{|c|c|c|c|}
\hline Variables & Synchronous metastases, $n=15$ & Metachronous metastases, $n=13$ & $p$ \\
\hline \multicolumn{4}{|l|}{ Type of hepatectomy } \\
\hline Partial hepatectomy & 12 & 7 & \multirow[t]{4}{*}{0.130} \\
\hline Subsegmentectomy & 1 & 1 & \\
\hline Segmentectomy & 1 & 5 & \\
\hline Right hepatectomy & 1 & 0 & \\
\hline Intraoperative blood loss $(\mathrm{ml})^{\mathrm{a}}$ & $670(186-1870)$ & $397(92-1682)$ & 0.185 \\
\hline \multicolumn{4}{|l|}{ Intraoperative blood transfusion } \\
\hline Performed & 4 & 1 & \multirow[t]{2}{*}{0.33} \\
\hline Not performed & 11 & 12 & \\
\hline \multicolumn{4}{|l|}{ Postoperative complications ${ }^{\mathrm{b}}$} \\
\hline Absent & 5 & 12 & \multirow[t]{10}{*}{0.002} \\
\hline Present & 10 & 1 & \\
\hline Intraabdominal abscess & 3 & 0 & \\
\hline Anastomotic leakage & 2 & 0 & \\
\hline Bile leakage & 2 & 0 & \\
\hline Cholangitis & 2 & 0 & \\
\hline Wound infection & 1 & 0 & \\
\hline Chylorrhea & 1 & 0 & \\
\hline Peripheral catheter infection & 1 & 0 & \\
\hline Hepatic infraction & 0 & 1 & \\
\hline \multicolumn{4}{|l|}{ Number of liver metastases } \\
\hline Solitary & 8 & 12 & \multirow[t]{2}{*}{0.038} \\
\hline Multiple & 7 & 1 & \\
\hline Postoperative mortality & 0 & 0 & - \\
\hline Postoperative hospital stay (days) ${ }^{\mathrm{a}}$ & $16(7-34)$ & $9(7-13)$ & 0.003 \\
\hline
\end{tabular}

a Median (range)

${ }^{\mathrm{b}}$ Grade II or higher according to the Clavien-Dindo classification

large-scale multi-institutional retrospective cohort study with a large sample of 256 patients [15]. Furthermore, Takemura et al. also reported good results with a 5-year survival rate and MST of $37 \%$ and 34 months, respectively, using the same criteria for hepatectomy as ours, namely, three or fewer liver metastases [14].

The controversies of these reports may be partly because of the long accumulation period and various diagnostic modalities for liver metastasis. In the present study, we determined the criteria for surgery using modern diagnostic modalities. It has been reported that the diagnostic ability of contrast-enhanced MRI is more sensitive than that of conventional enhanced CT scan, particularly in the detection of small lesions. In this study, we occasionally observed that several metastases were detected using contrast-enhanced MRI that were not detected using a conventional CT scan, which only reported solitary metastases. Thus, small multiple metastases might have been present in previous studies that reported a survival advantage for the resection of solitary metastasis. Therefore, it is reasonable that relatively better survival was demonstrated in multiple metastases up to three lesions when diagnosis was made using sensitive contrast-enhanced MRI. We speculate that hepatectomy can be appropriately performed for as many as three metastatic lesions if diagnosis is made using modern technologies.

Although the multivariate analysis revealed no independent prognostic factors, the univariate analysis of survival-related factors showed that outcomes were poorer in the synchronous liver metastasis group. Ambiru et al. [10], Okano et al. [11], and Schildberg et al. [13] have also reported poorer survival outcomes in patients with synchronous liver metastases than in those with metachronous liver metastases. For liver metastases of colorectal cancer, Tsai et al. [22] reported that patients with synchronous liver metastases who underwent resection are likely to have a greater number of metastases. Generally, they have metastases in both lobes and a shorter relapse-free survival time compared with patients with metachronous liver metastases. Although the biological differences between synchronous and metachronous metastases of gastric cancer remain unknown, the present study also found that 


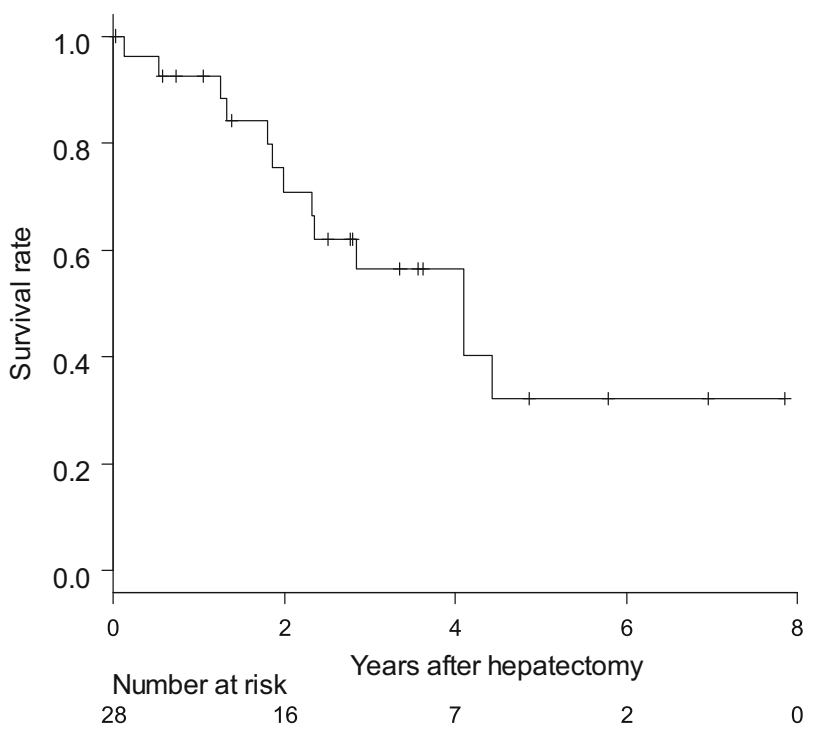

Fig. 1 Kaplan-Meier analysis of overall survival in 28 patients who underwent hepatectomy for liver metastases of gastric cancer. The 5 -year survival rate was $32 \%$

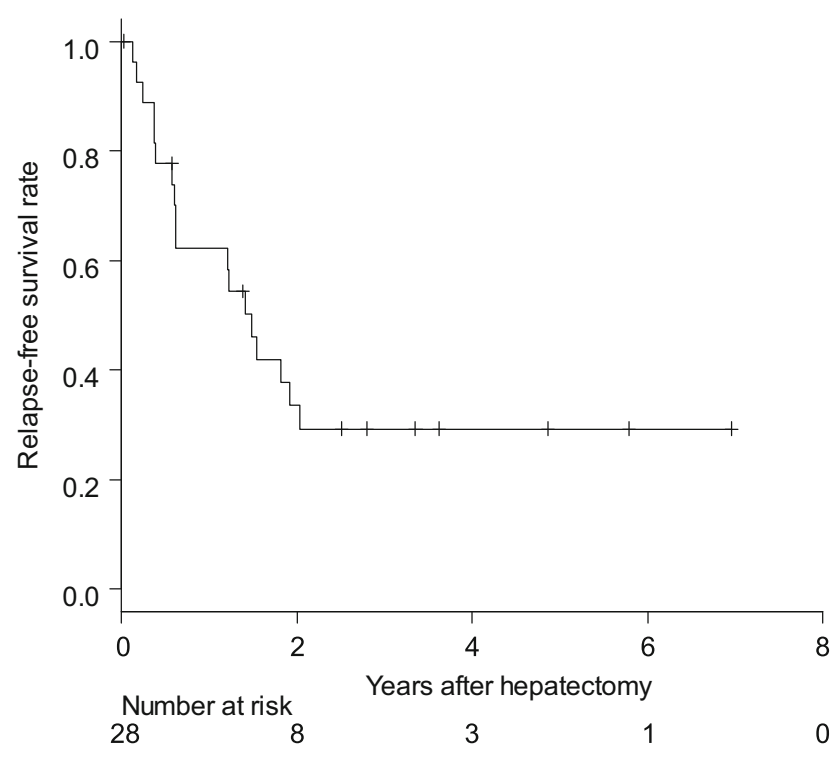

Fig. 2 Kaplan-Meier analysis of relapse-free survival in 28 patients who underwent hepatectomy for liver metastases of gastric cancer. The 5-year relapse-free survival rate was $29 \%$

many cases of resectable synchronous metastasis had multiple metastases. This finding suggests that synchronous liver metastases may be more malignant than metachronous LMGC.

Differences were seen in survival outcomes depending on the presence or absence of postoperative complications. In various diseases, postoperative complications have been reported to exacerbate survival outcomes. In a previous paper [23], we reported the association between
Table 3 Univariate analysis of factors affecting survival

\begin{tabular}{|c|c|c|c|c|}
\hline Variables & $n$ & HR & $95 \% \mathrm{CI}$ & $p$ \\
\hline \multicolumn{5}{|c|}{ Age at the time of hepatectomy } \\
\hline$<70$ years & 12 & 1.000 & & \multirow[t]{2}{*}{0.030} \\
\hline$\geq 70$ years & 16 & 3.845 & $1.137-13.010$ & \\
\hline \multicolumn{5}{|l|}{ Sex } \\
\hline Male & 23 & 1.000 & & \multirow[t]{2}{*}{0.318} \\
\hline Female & 5 & 0.353 & $0.0457-2.727$ & \\
\hline \multicolumn{5}{|l|}{ Liver metastases } \\
\hline \multicolumn{5}{|c|}{ Timing of liver metastases } \\
\hline Metachronous & 13 & 1.000 & & \multirow[t]{2}{*}{0.017} \\
\hline Synchronous & 15 & 4.985 & $1.337-18.590$ & \\
\hline \multicolumn{5}{|c|}{ Number of liver metastases } \\
\hline Solitary & 20 & 1.000 & & \multirow[t]{2}{*}{0.070} \\
\hline Multiple & 8 & 2.767 & $0.918-11.840$ & \\
\hline \multicolumn{5}{|c|}{ Intraoperative blood transfusion } \\
\hline Not performed & 23 & 1.000 & & \multirow[t]{2}{*}{0.067} \\
\hline Performed & 5 & 3.298 & $0.880-10.500$ & \\
\hline \multicolumn{5}{|c|}{ Maximum tumor size } \\
\hline$<30 \mathrm{~mm}$ & 15 & 1.000 & & \multirow[t]{2}{*}{0.518} \\
\hline$\geq 30 \mathrm{~mm}$ & 13 & 0.687 & $0.220-2.145$ & \\
\hline \multicolumn{5}{|c|}{ Postoperative complications ${ }^{\mathrm{c}}$} \\
\hline Absent & 17 & 1.000 & & \multirow[t]{2}{*}{0.042} \\
\hline Present & 11 & 3.423 & $1.045-11.210$ & \\
\hline
\end{tabular}

Adjuvant chemotherapy after hepatectomy

$$
\begin{array}{llll}
\text { Not performed } & 16 & 1.000 & \\
\text { Performed } & 12 & 0.780 & 0.256-2.380
\end{array}
$$

Primary tumor

Maximum tumor size (mm)

$$
\begin{array}{crrr}
<50 \mathrm{~mm} & 10 & 1.000 & \\
\geq 50 \mathrm{~mm} & 18 & 1.240 & 0.380-4.043 \\
\text { Histological differentiation } & & \\
\quad \text { Differentiated } & 22 & 1.000 & \\
\text { Undifferentiated } & 6 & 0.303 & 0.039-2.336 \\
\text { Pathological T classification }(\mathrm{pT}) & \\
\text { pT1, pT2 } & 20 & 1.000 & \\
\text { pT3, pT4 } & 8 & 0.788 & 0.203-3.066
\end{array}
$$

$\begin{array}{lrrr}\text { Differentiated } & 22 & 1.000 & \\ \text { Undifferentiated } & 6 & 0.303 & 0.039-2.336\end{array}$

Pathological N classification $(\mathrm{pN})$

$$
\begin{array}{lrll}
\text { pN0 } & 3 & 1.000 & \\
\text { pN1, pN2, pN3 } & 25 & >10 & 0.000-\propto
\end{array}
$$

Lymphatic invasion

$$
\begin{array}{lrrr}
\text { Negative } & 8 & 1.000 & \\
\text { Positive } & 20 & 2.571 & 0.559-11.830
\end{array}
$$

Venous invasion

$\begin{array}{lrrr}\text { Negative } & 4 & 1.000 & \\ \text { Positive } & 24 & 0.845 & 0.187-3.840\end{array}$

$H R$ hazard ratio, $C I$ confidence index

a Grade II or higher according to the Clavien-Dindo classification 


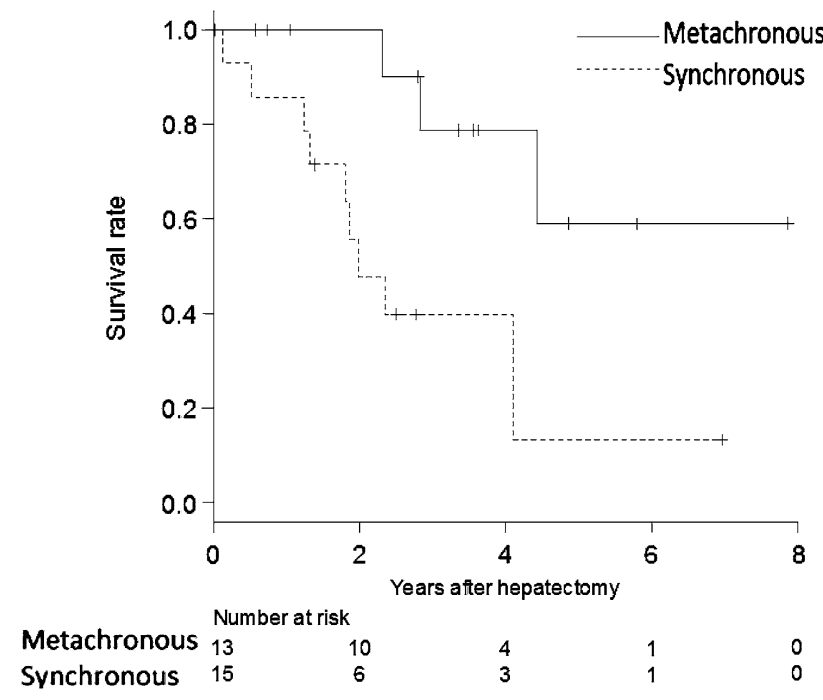

Fig. 3 The 5-year survival rates of patients with metachronous liver metastases $(59 \%)$ or synchronous liver metastases $(13 \%)$. There was a significant difference between the groups (log-rank test, $p=0.009$ )

intraabdominal infectious complications after surgery for gastric cancer and survival outcomes. Hayashi et al. [24] and Kubota et al. [25] also reported that complications following surgery for gastric cancer shorten relapse-free and overall survival rates. In the present study, survival outcomes may have been affected by the increased complications resulting from the concurrent performance of gastrectomy and hepatectomy.

Elder patients also demonstrated poor survival in the present study, in contrast to previous reports. The possible reason is that the average age was relatively higher in the present study than in previous studies. In addition, in younger patients (less than 70 years old), all of 6 patients who recurred after hepatectomy received chemotherapy or repeat hepatectomy, whereas 6 of 11 elder patients (70 years old or more) who recurred did not receive active treatment. This difference in treatment modality after recurrence may cause the difference of survival between elder patients and non-elder patients.

Liver recurrence in $15(54 \%)$ of the 28 patients highlighted the importance of controlling the high liver recurrence rate after hepatectomy; however, no established treatment strategy is available for recurrence in the liver remnant. Takemura et al. [26] reported a 5-year survival rate and MST of $47 \%$ and 31 months, respectively, in 14 patients who underwent repeat hepatectomy for recurrence in the liver remnant. Two of the three patients who underwent repeat hepatectomy in our study also achieved long-term survival, suggesting that resection may be considered if the aforementioned indications are met.

The usefulness of radiofrequency ablation (RFA) had been also demonstrated as a local therapy for liver metastasis. Guner et al. compared the results for LMGC between surgery and RFA and reported that similar results were obtained in the surgery group and RFA group [27]. RFA may be considered as a treatment option in patients for whom surgery is difficult for certain reasons even if the number of metastases is small.

This study had some limitations. First, it was based on retrospective analysis of a small sample size from a single institution, and multivariate analysis could not identify any independent prognostic factors. Most previous studies of hepatectomy for patients with LMGC were performed at a single institute over a relatively long period. Conversely, increased diagnostic ability such as contrast-enhanced MRI using liver-specific contrast agent has allowed detection of small metastases that were not previously detected. The results of this study are derived from successive cases treated over a short period under a fixed diagnostic modality and treatment policy. Therefore, this study, based on current diagnostic methods, is significant in that we were able to demonstrate good treatment outcomes in patients with three or fewer liver metastases. Second, the present study included some patients with a short follow-up period owing to the small number of patients who met the eligibility criteria during the 10 years of this study in a single institution. Further patient accumulation and followup are needed to demonstrate the true benefits of surgical therapy for LMGC.

In conclusion, hepatectomy has significance if indications are appropriately limited, particularly in cases of metachronous liver metastases, where active resection should be considered. However, treatment selection by modern diagnostic methods such as contrast-enhanced MRI using liver-specific contrast agent and improved surgical techniques to prevent postoperative complications are required.

Acknowledgments This research was supported by a grant from the Practical Research for Innovative Cancer Control (\#15ck0106043h0002) from the Japan Agency for Medical Research and Development, AMED.

\section{Compliance with ethical standards}

Conflict of interest The authors declare that they have no conflicts of interest.

Ethical standards All procedures followed were in accordance with the ethical standards of the responsible committee on human experimentation (institutional and national) and with the Helsinki Declaration of 1964 and later versions. Informed consent or an appropriate substitute for it was obtained from all patients included in the study.

\section{References}

1. Moon YW, Jeung HC, Rha SY, Yoo NC, Roh JK, Noh SH, et al. Changing patterns of prognosticators during 15 -year follow-up of 
advanced gastric cancer after radical gastrectomy and adjuvant chemotherapy: a 15-year follow-up study at a single Korean institute. Ann Surg Oncol. 2007;14:2730-7.

2. Yoo CH, Noh SH, Shin DW, Choi SH, Min JS. Recurrence following curative resection for gastric carcinoma. $\mathrm{Br} \mathrm{J}$ Surg. 2000;87:236-42.

3. Sakuramoto S, Sasako M, Yamaguchi T, Kinoshita T, Fujii M, Nashimoto A, et al. Adjuvant chemotherapy for gastric cancer with S-1, an oral fluoropyrimidine. $\mathrm{N}$ Engl $\mathrm{J}$ Med. 2007;357:1810-20.

4. Ohtsu A, Shah MA, Van Cutsem E, Rha SY, Sawaki A, Park SR, et al. Bevacizumab in combination with chemotherapy as firstline therapy in advanced gastric cancer: a randomized, doubleblind, placebo-controlled phase III study. J Clin Oncol. 2011;29:3968-76.

5. Bang YJ, Van Cutsem E, Feyereislova A, Chung HC, Shen L, Sawaki A, et al. Trastuzumab in combination with chemotherapy versus chemotherapy alone for treatment of HER2-positive advanced gastric or gastro-oesophageal junction cancer (ToGA): a phase 3, open-label, randomised controlled trial. Lancet. 2010;376:687-97.

6. Watanabe T, Itabashi M, Shimada Y, Tanaka S, Ito Y, Ajioka Y, et al. Japanese Society for Cancer of the Colon and Rectum (JSCCR) Guidelines 2014 for treatment of colorectal cancer. Int J Clin Oncol. 2015;20:207-39.

7. Abdalla EK, Vauthey JN, Ellis LM, Ellis V, Pollock R, Broglio $\mathrm{KR}$, et al. Recurrence and outcomes following hepatic resection, radiofrequency ablation, and combined resection/ablation for colorectal liver metastases. Ann Surg. 2004;239:818-25 (discussion 25-27).

8. Fernandez FG, Drebin JA, Linehan DC, Dehdashti F, Siegel BA, Strasberg SM. Five-year survival after resection of hepatic metastases from colorectal cancer in patients screened by positron emission tomography with F-18 fluorodeoxyglucose (FDGPET). Ann Surg. 2004;240:438-47 (discussion 47-50).

9. Marrelli D, Roviello F, De Stefano A, Fotia G, Giliberto C, Garosi L, et al. Risk factors for liver metastases after curative surgical procedures for gastric cancer: a prospective study of 208 patients treated with surgical resection. J Am Coll Surg. 2004;198:51-8.

10. Ambiru S, Miyazaki M, Ito H, Nakagawa K, Shimizu H, Yoshidome $\mathrm{H}$, et al. Benefits and limits of hepatic resection for gastric metastases. Am J Surg. 2001;181:279-83.

11. Okano K, Maeba T, Ishimura K, Karasawa Y, Goda F, Wakabayashi $\mathrm{H}$, et al. Hepatic resection for metastatic tumors from gastric cancer. Ann Surg. 2002;235:86-91.

12. Garancini M, Uggeri F, Degrate L, Nespoli L, Gianotti L, Nespoli A, et al. Surgical treatment of liver metastases of gastric cancer: is local treatment in a systemic disease worthwhile? HPB (Oxford). 2012;14:209-15.

13. Schildberg CW, Croner R, Merkel S, Schellerer V, Muller V, Yedibela $\mathrm{S}$, et al. Outcome of operative therapy of hepatic metastatic stomach carcinoma: a retrospective analysis. World $\mathrm{J}$ Surg. 2012;36:872-8.
14. Takemura N, Saiura A, Koga R, Arita J, Yoshioka R, Ono Y, et al. Long-term outcomes after surgical resection for gastric cancer liver metastasis: an analysis of 64 macroscopically complete resections. Langenbecks Arch Surg. 2012;397:951-7.

15. Kinoshita T, Kinoshita T, Saiura A, Esaki M, Sakamoto H, Yamanaka T. Multicentre analysis of long-term outcome after surgical resection for gastric cancer liver metastases. Br J Surg. 2015;102(1):102-7.

16. Tsurusaki M, Sofue K, Murakami T. Current evidence for the diagnostic value of gadoxetic acid-enhanced magnetic resonance imaging for liver metastasis. Hepatol Res. 2016. doi:10.1111/ hepr.12646.

17. Japanese classification of gastric carcinoma. 3rd English edition. Gastric Cancer. 2011;14:101-12.

18. Clavien PA, Barkun J, de Oliveira ML, Vauthey JN, Dindo D, Schulick RD, et al. The Clavien-Dindo classification of surgical complications: five-year experience. Ann Surg. 2009;250: 187-96.

19. Kanda Y. Investigation of the freely available easy-to-use software 'EZR' for medical statistics. Bone Marrow Transplant. 2013;48:452-8.

20. Oki E, Tokunaga S, Emi Y, Kusumoto T, Yamamoto M, Fukuzawa K, et al. Surgical treatment of liver metastasis of gastric cancer: a retrospective multicenter cohort study (KSCC1302). Gastric Cancer. 2015. doi:10.1007/s10120-015-0530-z.

21. Fujitani K, Yang HK, Mizusawa J, Kim YW, Terashima M, Han SU, et al. Gastrectomy plus chemotherapy versus chemotherapy alone for advanced gastric cancer with a single non-curable factor (REGATTA): a phase 3, randomised controlled trial. Lancet Oncol. 2016;17:309-18.

22. Tsai MS, Su YH, Ho MC, Liang JT, Chen TP, Lai HS, et al. Clinicopathological features and prognosis in resectable synchronous and metachronous colorectal liver metastasis. Ann Surg Oncol. 2007;14:786-94.

23. Tokunaga M, Tanizawa Y, Bando E, Kawamura T, Terashima M. Poor survival rate in patients with postoperative intra-abdominal infectious complications following curative gastrectomy for gastric cancer. Ann Surg Oncol. 2013;20:1575-83.

24. Hayashi T, Yoshikawa T, Aoyama T, Hasegawa S, Yamada T, Tsuchida $\mathrm{K}$, et al. Impact of infectious complications on gastric cancer recurrence. Gastric Cancer. 2015;18:368-74.

25. Kubota T, Hiki N, Sano T, Nomura S, Nunobe S, Kumagai K, et al. Prognostic significance of complications after curative surgery for gastric cancer. Ann Surg Oncol. 2014;21:891-8.

26. Takemura N, Saiura A, Koga R, Yoshioka R, Yamamoto J, Kokudo N. Repeat hepatectomy for recurrent liver metastasis from gastric carcinoma. World J Surg. 2013;37:2664-70.

27. Guner A, Son T, Cho I, Kwon IG, An JY, Kim HI, et al. Liverdirected treatments for liver metastasis from gastric adenocarcinoma: comparison between liver resection and radiofrequency ablation. Gastric Cancer. 2015. doi:10.1007/s10120-015-0522-z. 